\title{
Special Issue on Open Source Software-Supported Robotics Research
}

\author{
Caroline Pantofaru • Sachin Chitta • Brian Gerkey • \\ Radu Rusu • William D. Smart • Richard Vaughan
}

Received: 16 December 2012 / Accepted: 5 January 2013 / Published online: 7 February 2013

(C) Springer Science+Business Media New York 2013

Historically, robotics research has been disseminated through academic papers which lacked important implementation details, or one-time demonstrations of functionality. Neither of these methods has been conducive to either repeatable science, nor to building upon previous work. A shift is happening, however. The increasing distribution and use of open source software within robotics is moving the field to a model in which code is distributed, repeatedly executed and built upon. This cultural shift has the potential to accelerate robotics development by encouraging robust algorithms, algorithm comparison, and collaborations between research groups. Additionally, this shift should prevent new graduate students from reimplementing common algorithms. This special issue of Autonomous Robots is dedicated to exploring the use of open source software within a research context.

Creating and disseminating usable software is timeconsuming. It involves re-writing prototype software to be

C. Pantofaru $(\varangle) \cdot$ S. Chitta

Willow Garage, Inc., Menlo Park, CA, USA

e-mail: pantofaru@willowgarage.com

S. Chitta

e-mail: sachinc@willowgarage.com

B. Gerkey

Open Source Robotics Foundation, Mountain View, CA, USA

e-mail: gerkey@osrfoundation.org

R. Rusu

Open Perception, Menlo Park, CA, USA

e-mail: rusu@ openperception.org

W. D. Smart

Oregon State University, Corvallis, OR, USA

e-mail: smartw@engr.orst.edu

R. Vaughan

Simon Fraser University, Burnaby, BC, Canada

e-mail:vaughan@sfu.ca efficient, structuring it to be readable and extendable, and testing it thoroughly. It also involves time spent writing documentation, and not to be forgotten, time spent supporting the community using the software. The benefits, however, are valuable. The creators enjoy wider visibility and credit than a paper alone could offer, and often receive interesting ideas for extending their work. The users receive building blocks for their own systems and role models for their research.

Although the value of distributing reusable software is now being recognized, it rarely results in academic publications, and so has become a secondary pursuit for the academic community. This issue aims to stress the importance of disseminating quality software components. In this issue, we invite you to focus on software contributions alongside algorithmic advances, and reflect on how the availability of software is helping the field progress. The papers in this issue represent multi-faceted contributions, being evaluated on both their theoretical research contributions as well as the availability and usability of their code. We hope that you will find the software contributions useful for your own research.

A brief overview of each paper follows.

The paper by Pomerleau et al., "Comparing ICP Variants on Real-World Data Sets: Open-source library and experimental protocol," covers the range of contributions for this journal issue. Pomerleau et al. look at the ICP algorithm which is so important and so often used in robotics systems, but whose implementations are difficult to compare due to a lack of standardized interfaces. This paper presents an opensource library implementation of ICP which is fast yet modular, and thus adaptable for a range of robotics applications. In addition, this paper presents a framework for comparing multiple ICP implementations and provides a comparison of two baseline ICP implementations.

Ball et al. take a different view on SLAM in "OpenRatSLAM: An Open Source Brain-Based SLAM System," by 
taking inspiration from rodent neural processes underlying navigation. They have created a system built atop ROS that navigates via imagery from a monocular camera. Successful experiments include delivery tasks and mapping a suburb, as well as demonstrations on two publicly available datasets.

Dryanovski et al. take indoor navigation off the ground in "An Open-Source Navigation System for Micro-Air Vehicles". This paper provides a modular system for quadrotor navigation that builds upon the ROS infrastructure. Keeping in mind code reuse, the system provides control, state estimation, path-planning and teleoperation, but interfaces with the ROS 2D SLAM and 3D mapping tools. At the same time, the software runs onboard in real time, making it useful in practice.

Navigation and mapping frameworks rely on an underlying efficient representation of data such as occupancy maps. "OctoMap: An Efficient Probabilistic 3D Mapping Framework Based on Octrees" by Hornung et al. provides exactly that. Currently in use by many researchers, the OctoMap framework provides functionality for efficiently storing and accessing 3D data using the octree data structure.

Shifting gears, Romano et al., look at the underused sense of sound in "ROS Open-source Audio Recognizer: ROAR". Although speech recognition has flourished recently, sound classification for other tasks has not been so readily adopted. Sound, however, can give a wealth of cues about the results of a robot's actions in the world. For example, a crash might tell us that the robot has hit something or dropped an object it was holding. This paper provides a library known as ROAR containing tools for the learning and classification of audio events.

In "The ManyEars Open Framework - Microphone Array Open Software and Open Hardware System for Robotic Application," Grondin et al. also recognize the importance of sound, and note that a critical cue is the direction in which the sound originated. They provide the ManyEars library, built atop ROS, for localizing, tracking and separating sounds. They additionally provide a customized microphone board and sound card as open hardware for use on various robots.

Finally, the paper by Valero-Gomez et al., "A new Paradigm for Open Robotics Research and Education with the C++ OOML," tackles the hardware domain. An array of accessible hardware platforms, such as the Arduino and 3D printer, have come onto the market recently. Software tools are required to make use of these platforms, and open source software tools will facilitate sharing designs. This paper presents an open source mechanics library which allows users to model hardware through object-oriented $\mathrm{C}++$ code, and generates fabrication files.

These papers reflect the growing trend in the research community toward reusable, open software and even hardware. Hopefully this issue will expose you to new research and software that you can use in your own robot systems. Enjoy the issue!

\section{Author Biographies}

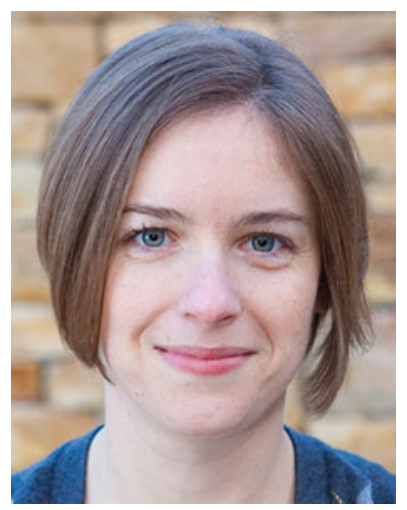

Caroline Pantofaru is a Research Scientist at Willow Garage, Inc. She holds a PhD and MS in Robotics from The Robotics Institute, Carnegie Mellon University, and an Honours BSc in Computer Science and Mathematics from The University of Toronto. Her primary research interests are in the field of computer vision, including object recognition, people detection and tracking, and scene understanding. She is also interested in human-robot interaction. Dr. Pantofaru has been an Associate Editor and committee member for numerous top-tier robotics and computer vision conferences, as well as organized multiple workshops at these meetings. She has encouraged open robotics research by leading the PR2 Beta Program and community.

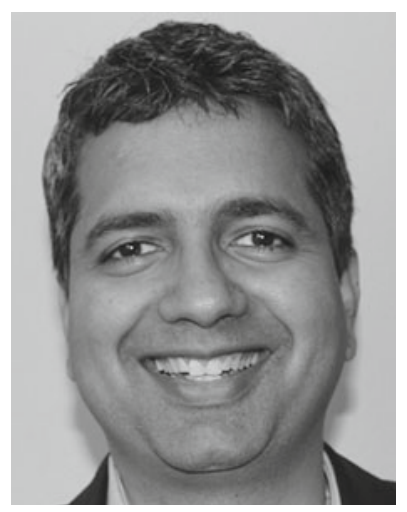

Sachin Chitta is the manager of the Autonomous Mobile Manipulation Group at Willow Garage, Inc. As a Research Scientist at Willow Garage since 2007, he led the development of mobile manipulation capabilities in ROS including the arm navigation and 3D navigation set of stacks, leading to new applications in mobile pick and place, teaching robots new skills and navigation in unstructured environments. His current research interests are in low-cost hardware, motion planning, control, and sensing for mobile manipulation in unstructured environments. Dr. Chitta received his $\mathrm{PhD}$ in Mechanical Engineering from the University of Pennsylvania in 2005, and he was a postdoctoral researcher working on learning for quadruped locomotion and modular robotics in the GRASP Lab at Penn from 2005 to 2007.

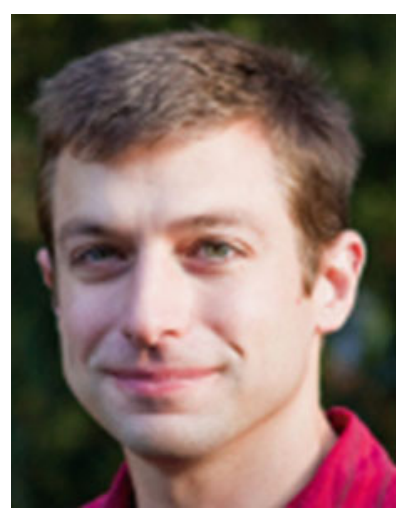

Brian Gerkey is CEO of OSRF. Prior to joining OSRF, Brian was Director of Open Source Development at Willow Garage. Previously, Brian was a Computer Scientist in the Artificial Intelligence Center at SRI, and before that, a postdoctoral research fellow in the Artificial Intelligence Lab at Stanford University. Brian received his $\mathrm{PhD}$ in Computer Science from the University of Southern California (USC) in 2003, his M.S. in Computer Science from USC in 2000 , and his B.S.E. in Computer Engineering, with a secondary major in 
Mathematics and a minor in Robotics and Automation, from Tulane University in 1998. Brian is a strong believer in, frequent contributor to, and constant beneficiary of open source software. Since 2008, Brian has worked on the ROS Project, which develops and releases one of the most widely used robot software platforms in robotics research and education (and soon industry). He is founding and former lead developer on the open source Player Project, which continues to maintain widely used robot simulation and development tools. For his work on Player and ROS, Brian was recognized by MIT Technology Review with the TR35 award in 2011.

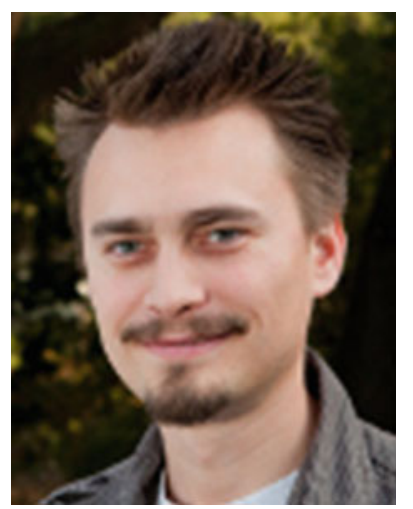

Radu Rusu is the President and CEO of Open Perception, Inc, a Visiting Lecturer at Stanford University, and a world renowned expert in $3 \mathrm{D}$ data processing with over 10 years of experience in the field. Before Open Perception, Radu was a Research Scientist at Willow Garage where he created the Point Cloud Library (PCL) project. Radu received his $\mathrm{PhD}$ in Computer Science from the Technische Universitaet Muenchen (TUM), Germany in 2009 with summa cum laude. During his PhD, Radu was affiliated with the CoTeSys (Cognition for Technical Systems) excellence cluster at TUM, the AIC (Artificial Intelligence Center) at SRI (Stanford Research Institute) as an International Fellow Researcher, and Willow Garage, working on 3D semantic mapping techniques for mobile robots. Over the past years, Radu has been on the board of many workshops and scientific events in the field, held at prestigious conferences such as: RSS, ICRA, IROS, ECCV, ICCV, AAAI, CVPR, 3DIMPVT, etc. He has co-authored over 70 peer-reviewed scientific publications, including 2 books, and three best paper awards and nominations (ICAR 2009, RSS 2011, and EURON George Giralt PhD award). Radu is a senior IEEE member, and the recipient of the IEEE RAS Early Career Award 2013. Radu also won the Open Source Software (OSS) World Challenge in 2011 with PCL.

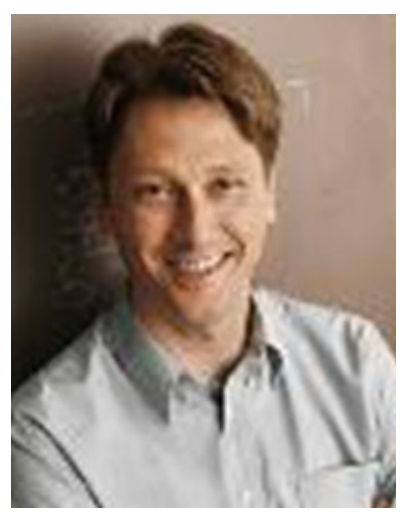

William D. Smart is an Associate Professor of Mechanical Engineering in the School of Mechanical, Industrial, and Manufacturing Engineering at Oregon State University. Before moving to OSU he spent 12 years on the faculty of the Department of Computer Science and Engineering at Washington University in St. Louis. He holds a $\mathrm{PhD}$ and MS in Computer Science from Brown University, and MSc in Intelligent Robotics from Edinburgh, and a BSc (hons) in Computer Science from Dundee University. His research interests cover the areas of human-robot interaction, mobile robotics, machine learning, and brain-computer interfaces.

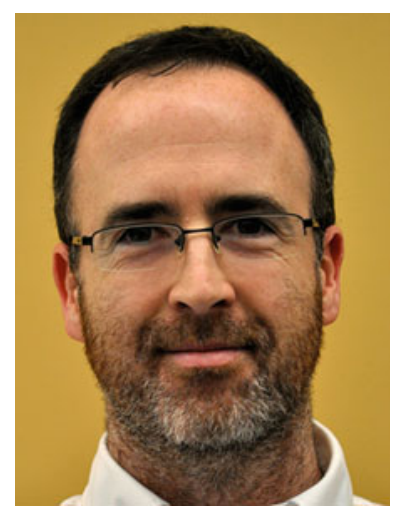

Richard Vaughan is Associate Professor and Director of Undergraduate Programs in the School of Computing Science at Simon Fraser University. His primary research group is the Autonomy Laboratory, which investigates the mechanisms of autonomous rational behaviour in machines and animals, with particular emphasis on managing energy and other resources. Vaughan's "Robot Sheepdog" was the first robot to control the behaviour of animals, and he recently demonstrated the first control of groups of robots by an uninstrumented human. Vaughan founded the Player/Stage Project with Brian Gerkey in 2000, and produced the most widely used and influential robotics development, networking and simulation platform of the last decade, downloaded over 260,000 times. Prof. Vaughan has held grants and contracts from NSERC, DRDC, DARPA, CFI and BCKDF and has served as Program Chair, Associate Editor and committee member for top-tier international conferences, and is Associate Editor for the journal Autonomous Robots. 